\title{
The Minimum Oracle Circuit Size Problem
}

\author{
Eric Allender ${ }^{1}$, Dhiraj Holden ${ }^{2}$, and Valentine Kabanets ${ }^{3}$ \\ 1 Department of Computer Science, Rutgers University \\ Piscataway, NJ, USA \\ allender@cs.rutgers.edu \\ 2 Department of Computer Science, California Institute of Technology \\ Pasadena, CA, USA \\ dholden@caltech.edu \\ 3 School of Computing Science, Simon Fraser University, Burnaby, BC, Canada \\ kabanets@cs.sfu.ca
}

\begin{abstract}
We consider variants of the Minimum Circuit Size Problem MCSP, where the goal is to minimize the size of oracle circuits computing a given function. When the oracle is QBF, the resulting problem MCSPQBF is known to be complete for PSPACE under ZPP reductions. We show that it is not complete under logspace reductions, and indeed it is not even hard for $\mathrm{TC}^{0}$ under uniform $\mathrm{AC}^{0}$ reductions. We obtain a variety of consequences that follow if oracle versions of MCSP are hard for various complexity classes under different types of reductions. We also prove analogous results for the problem of determining the resource-bounded Kolmogorov complexity of strings, for certain types of Kolmogorov complexity measures.
\end{abstract}

1998 ACM Subject Classification F.1.3 Complexity Measures and Classes

Keywords and phrases Kolmogorov complexity, minimum circuit size problem, PSPACE, NPintermediate sets

Digital Object Identifier 10.4230/LIPIcs.STACS.2015.21

\section{Introduction}

The Minimum Circuit Size Problem (MCSP) asks to decide, for a given truth table $f$ of a Boolean function and a parameter $s$, whether $f$ is computable by a Boolean circuit of size at most $s$. MCSP is a well-known example of a problem in NP that is widely believed to be intractable, although it is not known to be NP-complete. MCSP is known to be hard for the complexity class SZK under BPP-Turing reductions [4], which provides strong evidence for intractability. On the other hand, Kabanets and Cai showed [11] that if MCSP is NP-complete under the "usual" sort of polynomial-time reductions, then EXP $\nsubseteq \mathrm{P} /$ poly. This can not be interpreted as strong evidence against NP-completeness - since it is widely conjectured that EXP $\nsubseteq \mathrm{P} /$ poly - but it does indicate that it may be difficult to provide an NP-completeness proof.

However, there are other ways to define what the "usual" sort of reductions are: e.g., logspace, (uniform) $\mathrm{TC}^{0}, \mathrm{AC}^{0}$, or $\mathrm{NC}^{0}$. The overwhelming majority of problems that are known to be NP-complete are, in fact, NP-complete under very restricted kinds of reductions. Can we rule out NP-hardness of MCSP under such reductions?

Very recently, Murray and Williams [13] have shown that MCSP is not even P-hard under uniform NC ${ }^{0}$ reductions. Can MCSP be NP-hard under slightly stronger reductions, e.g., uniform $\mathrm{AC}^{0}$ reductions? We suspect that the answer is 'No', but so far we (like Murray

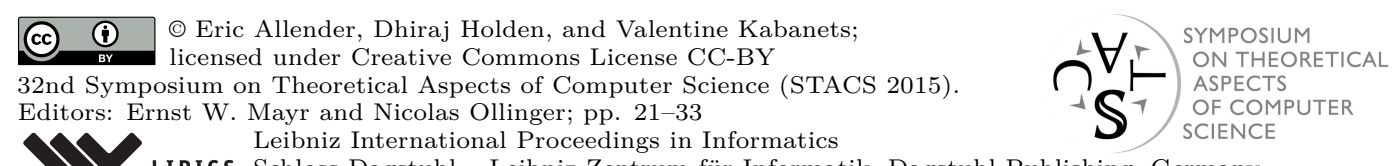


and Williams) can only show that P-hardness of MCSP under uniform $\mathrm{AC}^{0}, \mathrm{TC}^{0}$, or logspace reductions would imply new (likely) complexity lower bounds (in the spirit of [11]).

The main focus of the present paper is an oracle version of MCSP, denoted MCSP ${ }^{A}$ for a language $A$, which asks to decide for a given truth table $f$ and a parameter $s$ if $f$ is computable by an $A$-oracle circuit of size at most $s$. We prove a number of implications of hardness of $\mathrm{MCSP}^{A}$ for various choices of the oracle $A$, and various reductions. In particular, we prove for a PSPACE-complete $A$ that $\mathrm{MCSP}^{A}$ is not $\mathrm{P}$-hard under uniform $\mathrm{AC}^{0}$ reductions.

The results presented here (along with the results recently reported by Murray and Williams [13]) are the first results giving unlikely consequences that would follow if variants of MCSP or the various oracle circuit minimization problems are hard under a natural notion of reducibility. We also show that analogous results hold in the Kolmogorov complexity setting due to the correspondence between circuit size and Kolmogorov complexity, using the minimum-KT complexity problem defined in this paper.

Below we provide a summary of our main results.

\subsection{Our results}

Most of our results follow the template:

If $\mathrm{MCSP}^{A}$ is hard for a complexity class $\mathcal{C}$ under reductions of type $\mathcal{R}$, then complexity statement $\mathcal{S}$ is true.

Table 1 below states our results for different instantiations of $A, \mathcal{C}, \mathcal{R}$, and $\mathcal{S}$; note that $\mathcal{S}=\perp$ means that the assumption is false, i.e., $\operatorname{MCSP}^{A}$ is not $\mathcal{C}$-hard under $\mathcal{R}$-reductions. Throughout, we assume that the reader is familiar with complexity classes such as NP, PP, PSPACE, NEXP, etc. We denote the polynomial hierarchy by PH, and its linear-time version (linear-time hierarchy) by LTH. The Counting Hierarchy, denoted $\mathrm{CH}$, is the union of the classes PP, PP PP, etc.

Table 1 Summary of main results: If $\operatorname{MCSP}^{A}$ is $\mathcal{C}$-hard under $\mathcal{R}$, then $\mathcal{S}$. The last column shows the theorem where the result is stated in the paper.

\begin{tabular}{|c|c|c|c||c|}
\hline oracle $A$ & class $\mathcal{C}$ & reductions $\mathcal{R}$ & statement $\mathcal{S}$ & Theorem \\
\hline PH-hard & $\mathrm{TC}^{0}$ & uniform $\mathrm{AC}^{0}$ & $\perp$ & Theorem 20 \\
any & $\mathrm{TC}^{0}$ & uniform $\mathrm{AC}^{0}$ & $\mathrm{LTH} \not$ io-SIZE $^{A}\left[2^{\Omega(n)}\right]$ & Lemma 21 \\
any & $\mathrm{TC}^{0}$ & uniform $\mathrm{AC}^{0}$ & $\mathrm{NP}^{A} \nsubseteq \mathrm{SIZE}^{A}[$ poly $]$ & Corollary 24 \\
any in CH & $\mathrm{P}$ & uniform $\mathrm{TC}^{0}$ & $\mathrm{P} \neq \mathrm{PP}$ & Corollary 13 \\
$\emptyset$ & $\mathrm{P}$ & logspace & $\mathrm{P} \neq \mathrm{PSPACE}$ & Corollary 14 \\
QBF & $\mathrm{P}$ & logspace & $\mathrm{EXP}=\mathrm{PSPACE}$ & Corollary 18 \\
QBF & $\mathrm{NP}$ & logspace & NEXP $=$ PSPACE & Theorem 17 \\
QBF & PSPACE & logspace & $\perp$ & Corollary 19 \\
EXP-complete & $\mathrm{NP}$ & polytime & NEXP $=$ EXP & Theorem 15 \\
\hline
\end{tabular}

For the most restricted reductions, uniform $\mathrm{AC}^{0}$, we get that $\mathrm{MCSP}^{A}$ is not $\mathrm{TC}^{0}$-hard for any oracle $A$ such that $\mathrm{PH} \subseteq \mathrm{SIZE}^{A}[$ poly] (Theorem 20), e.g., for $A=\oplus \mathrm{P}$ (Corollary 23). For any oracle $A$, we conclude new circuit lower bounds for the linear-time hierarchy and for $\mathrm{NP}^{A}$ (Lemma 21 and Corollary $24^{1}$ ).

1 Prior to our work, Murray and Williams have shown that if $\mathrm{SAT} \leq{ }_{m}^{\mathrm{AC}}{ }^{0} \mathrm{MCSP}$, then NP $\nsubseteq \mathrm{P} /$ poly [13]. Their result is similar to (and is implied by) our Corollary 24 for the case of $A=\emptyset$. 
If MCSP is $\mathrm{P}$-hard under uniform $\mathrm{TC}^{0}$ or logspace reductions, then $\mathrm{P}$ is different from PP or from PSPACE (Corollaries 13 and 14).

One of the more interesting oracle circuit minimization problems is MCSPQBF. It was shown in [3] that MCSPQBF is complete for PSPACE under ZPP-Turing reductions, but the question of whether it is complete for PSPACE under more restrictive reductions was left open. For most natural complexity classes $\mathcal{C}$ above PSPACE, there is a corresponding oracle circuit minimization problem (which we will sometimes denote MCSP $\mathcal{C}$ ) that is known to be complete under $\mathrm{P} /$ poly reductions, but is not known to be complete under more restrictive reductions [3]. For the particular case of $\mathcal{C}=$ PSPACE, we denote this as MCSPQBF. We show that MCSPQBF is not PSPACE-complete under logspace reductions (Corollary 19). Furthermore, it is not even $\mathrm{TC}^{0}$-hard under uniform $\mathrm{AC}^{0}$ reductions (Theorem 20).

Finally, for even more powerful oracles $A$, we can handle even general polynomial-time reductions. We show that if SAT $\leq_{m}^{p}$ MCSPEXP, then EXP $=$ NEXP (Theorem 15).

We believe that MCSP is not $\mathrm{TC}^{0}$-hard under even nonuniform $\mathrm{AC}^{0}$ reductions. While we are unable to prove this, we can rule out restricted $A C^{0}$ reductions for a certain gap version of MCSP. Define gap-MCSP as follows: Given a truth table $f$ and a parameter $s$, output 'Yes' if $f$ requires circuit size $s$, and output 'No' if $f$ can be computed by a circuit of size at most $s / 2$. Call a mapping from $n$-bit strings to $m$-bit strings $\alpha(n)$-stretching if $m \leq n \cdot \alpha(n)$, for some function $\alpha: \mathbb{N} \rightarrow \mathbb{R}^{\geq 0}$.

We prove that gap-MCSP is not $\mathrm{TC}^{0}$-hard under nonuniform $\mathrm{AC}^{0}$ reductions that are $n^{1 / 31}$-stretching (Theorem 27).

\subsection{Related work}

The most closely related is the recent paper by Murray and Williams [13], which also considers the question whether MCSP is NP-complete under weak reductions, and proves a number of conditional and unconditional results. The main unconditional result is that MCSP is not $\mathrm{TC}^{0}$-hard under uniform $\mathrm{NC}^{0}$ reductions (or more generally, under $O\left(n^{1 / 2-\epsilon}\right)$-time projections, for every $\epsilon>0$ ); we give an alternative proof of this result (Theorem 25). For conditional results, [13] shows that if MCSP is NP-hard under uniform $\mathrm{AC}^{0}$ reductions, then $\mathrm{NP} \not \subset \mathrm{P} /$ poly and $\mathrm{E} \not \subset$ io-SIZE $\left[2^{\Omega(n)}\right]$ (also implied by our Corollary 24 and Lemma 21 ), and that NP-hardness of MCSP under general polynomial-time reductions implies EXP $\neq$ ZPP.

MCSP, MCSPQBF and other oracle circuit minimization problems are closely related to notions of resource-bounded Kolmogorov complexity. Briefly, a small (oracle) circuit is a short description of the string that represents the truth-table of the function computed by the circuit. Notions of resource-bounded Kolmogorov complexity were presented and investigated in [3] that are roughly equivalent to (oracle) circuit size.

In particular, there is a space-bounded notion of Kolmogorov complexity, KS, such that the set of KS-random strings (denoted $R_{\mathrm{KS}}$ ) is complete for PSPACE under ZPP reductions. It is shown in [3] that $R_{\mathrm{KS}}$ is not even hard for $\mathrm{TC}^{0}$ under $\mathrm{AC}^{0}$ reductions, and $R_{\mathrm{KS}}$ is not hard for PSPACE under logspace-Turing reductions. The proof of this non-hardness result also carries over to show that a set such as $\{f: f$ is the truth table of a function on $n$ variables that has $\mathrm{QBF}$ circuits of size at most $\left.2^{n / 2}\right\}$ is also not hard for $\mathrm{TC}^{0}$ under $\mathrm{AC}^{0}$ reductions, and is not hard for PSPACE under logspace-Turing reductions. However it does not immediately carry over to MCSPQBF, which is defined as $\{(f, i): f$ is the truth table of a function on $n$ variables that has QBF circuits of size at most $i\}$; similarly it does not carry over to the set $\{(x, i): \mathrm{KS}(x) \leq i\}$. Also, the techniques presented in [3] have not seemed to provide any tools to derive consequences assuming completeness results for oracle circuit minimization problems for oracles less powerful than PSPACE. We should point out, 
however, that [3] proves a result similar to (and weaker than) our Lemma 21 in the context of time-bounded Kolmogorov complexity: if $R_{\mathrm{KT}}$ is $\mathrm{TC}^{0}$-hard under $\mathrm{AC}^{0}$ many-one reductions, then $\mathrm{PH} \not \subset \mathrm{SIZE}\left[2^{n^{o(1)}}\right]$.

\subsection{Our techniques}

To illustrate our proof techniques, let us sketch a proof of one of our results: If MCSP is P-hard under uniform logspace reductions, then P $\neq$ PSPACE (Corollary 14).

The proof is by contradiction. Suppose that $\mathrm{P}=$ PSPACE. Our logspace reduction maps $n$-bit instances of QBF to $n^{c}$-bit instances $(f, s)$ of MCSP so that each bit of $f$ is computable in $O(\log n)$ space.

1. Imagine that our reduction is given as input a succinct version of QBF, where some poly $(\log n)$-size circuit $D$ on each $\log n$-bit input $1 \leq i \leq n$ computes the $i$ th bit of the QBF instance. It is not hard to see that our reduction, given the circuit $D$, can compute each bit of $f$ in poly $(\log n)$ space. Thus the Boolean function with the truth table $f$ is computable by a PSPACE $=\mathrm{P}$ algorithm (which also has the circuit $D$ as an input). It follows that this function $f$ is computable by some polynomial-size Boolean circuit.

2. Next, since we know that $f$ has at most polynomial circuit complexity, to decide the MCSP instance $(f, s)$, we only need to consider the case where $s<$ poly (since for big values of $s$, the answer is 'Yes'). But deciding such MCSP instances (which we call succinct MCSP) is possible in $\Sigma_{2}^{p}$ : guess a circuit of size at most $s$, and verify that it agrees with the given polynomial-size circuit for $f$ on all inputs.

3. Finally, since $\Sigma_{2}^{p} \subseteq$ PSPACE $=\mathrm{P}$, we get that our succinct MCSP instances can be decided in $P$. The reduction from succinct QBF to succinct MCSP is also in PSPACE $=P$. Hence, succinct QBF is in P. But, succinct QBF is EXPSPACE-complete, and so we get the collapse $\operatorname{EXPSPACE}=\mathrm{P}$, contradicting the hierarchy theorems.

In step (1) of the sketched proof, the uniformity of an assumed reduction to MCSP is used to argue that the truth table $f$ produced by the reduction is in fact "easy" to compute uniformly. The uniform complexity of computing the function $f$ is roughly the "exponential" analogue of the uniform complexity of the reduction. For circuit classes such as $A C^{0}$ and $\mathrm{TC}^{0}$, we use the well-known connection between the "exponential" analog of uniform $\mathrm{AC}^{0}$ and $\mathrm{PH}$, and between the "exponential" analog of uniform $\mathrm{TC}^{0}$ and $\mathrm{CH}$.

We use the uniform easiness of the function $f$ to conclude that $f$ has small circuit complexity (and hence our reduction actually outputs instances of succinct MCSP). To get that conclusion, we need to assume (or derive) the collapse to $\mathrm{P} /$ poly of the uniform complexity class that contains $f$; in our example above, we got it from the assumption that PSPACE $=$ P.

Step (2) exploits the fact that succinct MCSP does not become "exponentially harder" (unlike the usual succinct versions of hard problems), but is actually computable in $\Sigma_{2}^{p}$.

In Step (3), we combine the algorithm for our reduction and the algorithm for succinct MCSP to get an "efficient" algorithm for the succinct version of the input problem (succinct QBF in our example). Since the succinct version of the input problem does become exponentially harder than its non-succinct counterpart, we get some impossible collapse (which can be disproved by diagonalization).

We use this style of proof for all our results involving reductions computable by uniform $\mathrm{TC}^{0}$ and above. However, for the case of uniform $\mathrm{AC}^{0}$ (and below), we get stronger results by replacing the diagonalization argument of Step (3) with the nonuniform $A^{0}$ circuit lower bound for PARITY [10]. 
Remainder of the paper. We state the necessary definitions and auxiliary results in Section 2. Our main results are proved in Section 3, and some generalizations are given in Section 4. We give concluding remarks in Section 5.

\section{Definitions}

- Definition 1. The minimum circuit size problem MCSP, as defined in [11], is defined as $\{(f, s) \mid f$ has circuits of size $s\}$, where $f$ is a string of length $2^{m}$ encoding the entire truth-table of some $m$-variate Boolean function. (Versions of this problem have been studied long prior to [11]. See [4, 17] for a discussion of this history.) We will also consider the analogous problem for circuits with oracles, the Minimum $A$-Circuit Size problem MCSP ${ }^{A}$, defined analogously, where instead of ordinary circuits, we use circuits that also have oracle gates that query the oracle $A$. When $A$ is a standard complete problem for some complexity class $\mathcal{C}$, we may refer to this as $\mathrm{MCSP}^{\mathcal{C}}$.

We will not need to be very specific about the precise definition of the "size" of a circuit. Our results hold if the "size" of a circuit is the number of gates (including oracle gates), or the number of "wires", or the number of bits used to describe a circuit in some standard encoding. It is perhaps worth mentioning that the different versions of MCSP that one obtains using these different notions of "size" are not known to be efficiently reducible to each other.

Circuit size relative to oracle $A$ is polynomially-related to a version of time-bounded Kolmogorov complexity, denoted $\mathrm{KT}^{A}$, which was defined and studied in [3].

Definition 2. $\mathrm{KT}^{A}(x)=\min \left\{|d|+t: \forall b \in\{0,1, *\} \forall i \leq|x|+1 U^{A}(d, i, b)\right.$ accepts in $t$ steps iff $\left.x_{i}=b\right\}$. Here, $U$ is some fixed universal Turing machine, which has random access to the oracle $A$ and to the input string (or "description") $d ; x_{i}$ denotes the $i$-th symbol of $x$, where $x_{|x|+1}=*$.

By analogy to $\mathrm{MCSP}^{A}$, we define the "minimum KT problem":

Definition 3. $\operatorname{MKTP}^{A}=\left\{(x, i) \mid \mathrm{KT}^{A}(x) \leq i\right\}$.

All of our results that deal with $\mathrm{MCSP}^{A}$ also apply to $\mathrm{MKTP}^{A}$.

We wish to warn the reader that one's intuition can be a poor guide, when judging how $\mathrm{MCSP}^{A}$ and $\mathrm{MCSP}^{B}$ compare to each other, for given oracles $A$ and $B$. For instance, it is known that MCSPSAT ZPP-Turing reduces to MCSP ${ }^{Q B F}$ [3], but no deterministic reduction is known. Similarly, no efficient reduction of any sort is known between MCSP and MCSPSAT . Some of our theorems derive consequences from the assumption that MCSPSAT is hard for some complexity class under $\mathrm{AC}^{0}$ reductions. Although one might suspect that this is a weaker hypothesis than assuming that MCSP is hard for the same complexity class under $A C^{0}$ reductions - certainly the best upper bound for MCSP ${ }^{S A}$ is worse than the best known upper bound for MCSP - nonetheless we are not able to derive the same consequences assuming only that MCSP is hard. For essentially all time- and space-bounded complexity classes $\mathcal{C}$ that contain PSPACE, MCSP $\mathcal{C}$ is complete for $\mathcal{C} /$ poly under $\mathrm{P} /$ poly reductions $[3,6]$, but uniform reductions are known only for two cases [3]: when $\mathcal{C}=$ PSPACE $(\mathrm{MCSPQBF}$ is complete for PSPACE under ZPP reductions) and when $\mathcal{C}=$ EXP (MCSPEXP is complete for EXP under NP-Turing reductions). 


\subsection{Succinct Problems}

The study of succinct encodings of computational problems was introduced by [9, 16], and has been studied since then by $[18,7]$, among others. Succinct encodings play an important role in the proofs of our main results.

- Definition 4. Given a language $L$, we define the succinct version of $L$ (denoted succ. $L$ ) to be the language $\{C \mid t t(C) \in L\}$ where $C$ is a Boolean Circuit and $\operatorname{tt}(C)$ is the truth-table for C.

It will be necessary for us to consider "succinctly-presented" problems, where the circuit that constitutes the succinct description is itself an oracle circuit:

- Definition 5. Given a language $L$ and an oracle $A$, we define the $A$-succinct version of $L$ (denoted $A$-succ. $L$ ) to be the language $\{C \mid t t(C) \in L\}$ where $C$ is a Boolean Circuit with oracle gates, and $t(C)$ is the truth-table for $C$, when it is evaluated with oracle $A$. If $A=\emptyset$, we denote this language as succ. $L$.

The typical situation that arises is that the succinct version of a problem $A$ has exponentially greater complexity than $A$. In particular, this happens when $A$ is complete for a complexity class under "logtime reductions".

- Definition 6. We say that a function $f$ can be computed in logarithmic time if there exists a random-access Turing machine that, given $(x, i)$, computes the $i$ th bit $f(x)$ in time $O(\log |x|)$.

Building on prior work of $[16,9,18]$, Balcázar, Lozano, and Torán presented a large list of complexity classes $\left(\mathcal{C}_{1}, \mathcal{C}_{2}\right)$, where $\mathcal{C}_{1}$ is defined in terms of some resource bound $B(n)$ and $\mathcal{C}_{2}$ is defined in the same way, with resource bound $B\left(2^{n}\right)$, such that if a set $A$ is complete for $\mathcal{C}_{1}$ under logtime reductions, then succ. $A$ is complete for $\mathcal{C}_{2}$ under polynomial-time many-one reductions [7].

Somewhat surprisingly, the complexity of succ.MCSP appears not to be exponentially greater than that of MCSP. (Related observations were made earlier by Williams [19].)

- Theorem 7. succ.MCSP $\in \Sigma_{2}^{p}$

Proof. We present an algorithm in $\Sigma_{2}^{p}$ that decides succ.MCSP. Given an instance of succinct MCSP $C$, note that $C \in$ succ.MCSP iff $z$ is a string of the form $(f, s) \in \operatorname{MCSP}$, where $z=t t(C)$. By definition, $|z|$ must be a power of 2 , say $|z|=2^{r}$, and $|f|$ must also be a power of 2 , say $|f|=2^{m}$ for some $m<r$. Note also that if $s>|f|=2^{m}$, then $(f, s)$ should obviously be accepted, since every $m$-variate Boolean function has a circuit of size $2^{m}$. To be precise, we will choose one particular convention for encoding the pair $(f, s)$; other reasonable conventions will also yield a $\Sigma_{2}^{p}$ upper bound. Let us encode $(f, s)$ as a string of length $2^{m+1}$, where the first $2^{m}$ bits give the truth table for $f$, and the second $2^{m}$ bits give $s$ in binary. Note that this means that $C$ has $m+1$ input variables, and hardwiring the high-order input bit of $C$ to 0 results in a circuit $C^{\prime}$ for $f$ (of size at most $|C|$ ).

Using this encoding, the "interesting" instances $(f, s)$ are of the form where the second half of the string is all zeros, except possibly for the low-order $m$ bits (encoding a number $s \leq 2^{m}=|f|$. The low-order $m$ bits can be computed deterministically in polynomial time, given $C$, by evaluating $C$ on inputs $1^{m+1-\log m} 0^{\log m}, 1^{m+1-\log m} 0^{-1+\log m} 1, \ldots, 1^{m+1}$. Let the number encoded by the low-order $m$ bits be $s^{\prime}$. Then $C$ (an encoding of $(f, s)$ ) is in succ.MCSP iff 
- there is some bit position $j$ corresponding to one of the high-order $2^{m}-m$ bits of $s$ such that $C(j)=1$, or

- there exists a circuit $D$ of size at most $s^{\prime}$ such that, for all $i, D(i)=C^{\prime}(i)$ and for all bit positions $j$ corresponding to one of the high-order $2^{m}-m$ bits of $s, C(j)=0$ (and thus $\left.s=s^{\prime}\right)$.

It is easily seen that this can be checked in $\Sigma_{2}^{p}$.

Because this proof relativizes, we obtain:

Corollary 8. Let $A$ and $B$ be oracles such that $B \leq_{T}^{p} A$. Then $B$-succ.MCSP ${ }^{A}$ is in $\left(\Sigma_{2}^{p}\right)^{A}$.

Proof. We use the same encoding as in Theorem 7. Thus, an oracle circuit $C$ encoding an instance $(f, s)$ (where $f$ is an $m$-ary function) has $m+1$ input variables, and hardwiring the high-order input bit of $C$ to 0 results in an oracle circuit $C^{\prime}$ (with oracle $B$ ) for $f$ (of size at most $|C|$ ). But if $B \leq_{T}^{p} A$, then this also gives us an oracle circuit $C^{\prime \prime}$ (with oracle $A$ ) for $f$ (of size at most $|C|^{k}$ for some $k$ ), where we can obtain $C^{\prime \prime}$ from $C$ in polynomial time.

Then $C$ (an encoding of $(f, s))$ is in $B$-succ.MCSP ${ }^{A}$ iff

- there is some bit position $j$ corresponding to one of the high-order $2^{m}-m$ bits of $s$ such that $C^{B}(j)=1$, or

- there exists a circuit $D$ of size at most $s^{\prime}$ such that, for all $i, D^{A}(i)=C^{\prime \prime} A(i)$ and for all bit positions $j$ corresponding to one of the high-order $2^{m}-m$ bits of $s, C^{B}(j)=0$ (and thus $s=s^{\prime}$ ).

It is easily seen that this can be checked in $\left(\Sigma_{2}^{p}\right)^{A}$.

An analogous result also holds for $\mathrm{MKTP}^{A}$.

- Theorem 9. Let $A$ and $B$ be oracles such that $B \leq_{T}^{p} A$. Then $B$-succ.MKTP ${ }^{A}$ is in $\left(\Sigma_{2}^{p}\right)^{A}$.

\subsection{Constant-Depth Reductions}

- Proposition 10. Suppose that $f$ is a uniform $\mathrm{AC}^{0}$ reduction from a problem $A$ to a problem $B$. Let $C$ be an instance of succ.A. Then, the language $\{(C, i) \mid$ the ith bit of $f(t t(C))$ is 1$\}$ is in LTH (the linear-time hierarchy).

Proof. Consider the unary version of the above language: $\left\{1^{(C, i)} \mid\right.$ the $i$ th bit of $f(t t(C))$ is $1\}$; we claim that this language is in uniform $\mathrm{AC}^{0}$. To see this, note that after computing the length of the input (in binary), and thus obtaining a description of $C$ (of length $\log n$ ), an $\mathrm{AC}^{0}$ algorithm can compute each bit of $\operatorname{tt}(C)$. For instance, the $i$ th bit of $\operatorname{tt}(C)$ can be computed by guessing a bit vector of length $\log n$ recording the value of each gate of $C$ on input $i$, and then verifying that all of the guessed values are consistent. Once the bits of $t t(C)$ are available, then the $\mathrm{AC}^{0}$ algorithm computes $f(t t(C))$.

The result is now immediate, from [5, Proposition 5], which shows that the rudimentary languages (that is, the languages in the linear-time version LTH of the polynomial-time hierarchy $\mathrm{PH}$ ) are precisely the sets whose unary encodings are in Dlogtime-uniform $\mathrm{AC}^{0}$.

By an entirely analogous argument, we obtain:

- Proposition 11. Suppose that $f$ is a uniform $\mathrm{TC}^{0}$ reduction from a problem $A$ to a problem $B$. Let $C$ be an instance of succ.A. Then, the language $\{(C, i) \mid$ the ith bit of $f(t t(C))$ is 1$\}$ is in $\mathrm{CH}$. 


\section{$3 \quad$ Main Results}

\subsection{Conditional collapses and separations of complexity classes}

Our first theorem shows that significant conclusions follow if MCSP is hard for $\mathrm{P}$ under $\mathrm{AC}^{0}$ reductions.

- Theorem 12. If there is any set $A$ in the polynomial hierarchy such that $\mathrm{MCSP}^{A}$ (or $\mathrm{MKTP}^{A}$ ) is hard for $\mathrm{P}$ under $\mathrm{AC}^{0}$ reductions, then $\mathrm{P} \neq \mathrm{NP}$.

Proof. We present only the proof for $\mathrm{MCSP}^{A}$; the proof for MKTP ${ }^{A}$ is identical. Suppose that $\mathrm{P}=\mathrm{NP}$ and $\mathrm{MCSP}^{A}$ is hard for $\mathrm{P}$ under $\mathrm{AC}^{0}$ reductions. Thus, there is a family $\left\{C_{n}\right\}$ of $\mathrm{AC}^{0}$ circuits reducing SAT to $\mathrm{MCSP}^{A}$, such that $C_{n}(\phi)=f(\phi)$, where $f$ is the reduction function and $\phi$ is an instance of SAT.

Now we claim that succ.SAT $\leq_{m}^{p}$ succ.MCSP $^{A}$. To see this, consider an instance $D$ of succ.SAT (that is, a circuit $D$ on $n$ variables that, when given input $i$, outputs the $i$ th bit of a SAT instance of size $2^{n}$ ). This problem has been shown to be complete for NEXP[15]. By Proposition 10, we have that the language $\{(D, i) \mid$ the $i$ th bit of $f(t t(D))$ is 1$\}$ is in $\mathrm{PH}$. By our assumption that $\mathrm{P}=\mathrm{NP}$, we have that this language is in $P$. Let $E_{m}$ be a family of circuits deciding this language. The function that takes input $D$ and outputs $E_{|(D, n)|}$ (with $D$ hardwired in) is a polynomial-time reduction from succ.SAT to succ.MCSP ${ }^{A}$, which is in $\left(\Sigma_{2}^{p}\right)^{A}$, by Corollary 8 . Since $A \in \mathrm{P}$ (by our assumption that $\mathrm{P}=\mathrm{NP}$ ), we have that $\mathrm{NEXP} \subseteq \mathrm{P}$, which is a contradiction.

- Corollary 13. If there is any set $A \in \mathrm{CH}$ such that $\mathrm{MCSP}^{A}$ (or $\mathrm{MKTP}^{A}$ ) is hard for $\mathrm{P}$ under $\mathrm{TC}^{0}$ reductions, then $\mathrm{P} \neq \mathrm{PP}$.

Due to space limitations, this proof and several others are omitted. A more complete version may be found on ECCC.

- Corollary 14. Suppose that MCSP (or MKTP) is hard for P under logspace many-one reductions. Then $\mathrm{P} \neq \mathrm{PSPACE}$.

- Theorem 15. Suppose that MCSP ${ }^{\mathrm{EXP}}$ is hard for NP under polynomial-time reductions. Then NEXP = EXP.

Proof. Let $f$ be the reduction taking an instance of SAT to an instance of MCSP ${ }^{\mathrm{EXP}}$. We construct a reduction from succ.SAT to $B$-succ.MCSP ${ }^{\mathrm{EXP}}$ for some $B \in \mathrm{EXP}$.

Consider the language $L=\left\{(C, i) \mid\right.$ the $i$ th bit of $f\left(\phi_{C}\right)$ is 1$\}$, where $\phi_{C}$ is the formula described by the circuit $C$, viewed as an instance of succ.SAT with $n$ input variables. We can decide $L$ in exponential time because we can write down $\phi_{C}$ in exponential time, and then we can compute $f\left(\phi_{C}\right)$ in exponential time because $f$ is a poly-time reduction on an exponentially large instance. Let $\left\{D_{m}\right\}$ be a family of oracle circuits for $L$, using an oracle for an EXP-complete language $B$. Thus the mapping $C \mapsto D_{|C|+n}$ is a polynomial-time reduction from succ.SAT to $B$-succ.MCSP ${ }^{\mathrm{EXP}}$, which is in $\left(\Sigma_{2}^{p}\right) \mathrm{EXP}=\mathrm{EXP}$ (see, e.g., $[6$, Theorem 24]), and thus EXP $=$ NEXP.

- Corollary 16. Consider Levin's time-bounded Kolmogorov complexity measure Kt [12]. Suppose that $\{(x, i): K t(x) \leq i\}$ is hard for NP under polynomial-time reductions. Then $\mathrm{NEXP}=\mathrm{EXP}$.

- Theorem 17. If MCSPQBF or MKTPQBF is hard for NP under logspace reductions, then $\mathrm{NEXP}=\mathrm{PSPACE}$. 
Corollary 18. If MCSPQBF (or MKTPQBF) is hard for $\mathrm{P}$ under logspace reductions, then $\mathrm{EXP}=\mathrm{PSPACE}$.

Proof. The proof is identical to the proof of the preceding theorem, with NP replaced by $P$, and with NEXP replaced by EXP.

If we carry out a similar argument, replacing NP with PSPACE, we obtain the contradiction EXPSPACE $=$ PSPACE, yielding the following.

- Corollary 19. Neither MCSPQBF nor MKTPQBF is hard for PSPACE under logspace reductions.

\subsection{Impossibility of uniform $A C^{0}$ reductions}

- Theorem 20. For any language $A$ that is hard for $\mathrm{PH}$ under $\mathrm{P} /$ poly reductions, $\mathrm{MCSP}^{A}$ is not hard for $\mathrm{TC}^{0}$ under uniform $\mathrm{AC}^{0}$ reductions.

The theorem will follow from the next lemma. Recall that LTH (linear-time hierarchy) stands for the linear-time version of the polynomial-time hierarchy $\mathrm{PH}$.

- Lemma 21. Suppose that, for some language $A, \mathrm{MCSP}^{A}$ is $\mathrm{TC}^{0}$-hard under uniform $\mathrm{AC}^{0}$ reductions. Then $\mathrm{LTH} \nsubseteq$ io-SIZE ${ }^{A}\left[2^{\Omega(n)}\right]$.

Proof. It is shown in [1, Theorems 5.1 and 6.2] that if a set is hard for any class $\mathcal{C}$ that is closed under $\mathrm{TC}^{0}$ reductions under uniform $\mathrm{AC}^{0}$ reductions, then it is hard under lengthincreasing (uniform $\mathrm{AC}^{0}$ )-uniform $\mathrm{NC}^{0}$ reductions. (Although Theorems 5.1 and 6.2 in [1] are stated only for sets that are complete for $\mathcal{C}$, they do hold also assuming only hardness [2], using exactly the same proofs.) Here, the notion " $\mathrm{AC}^{0}$-uniform $\mathrm{NC}^{0}$ " refers to $\mathrm{NC}^{0}$ circuits with the property that direct connection language $D C L=\left\{(n, t, i, j) \mid\right.$ gate $i$ of $F_{n}$ has type $t$ and has an edge leading from gate $j\}$ with $n$ in unary is in Dlogtime-uniform $\mathrm{AC}^{0}$.

Hence, if $\mathrm{MCSP}^{A}$ is hard for $\mathrm{TC}^{0}$ under uniform $\mathrm{AC}^{0}$ reductions, then we get that PARITY is reducible to $\mathrm{MCSP}^{A}$ under a length-increasing (uniform $A C^{0}$ )-uniform $N C^{0}$ reduction. Such a reduction $R$ maps PARITY instances $x \in\{0,1\}^{n}$ to $\operatorname{MCSP}^{A}$ instances $(f, s)$, where $f$ is the truth table of a Boolean function, $f \in\{0,1\}^{m}$, for some $m$ such that $n \leq m \leq n^{O(1)}$, and $0 \leq s \leq m$ is the size parameter in binary, and hence $|s| \leq O(\log n)$.

Being the output of an $\mathrm{NC}^{0}$ reduction, the binary string $s$ depends on at most $O(\log n)$ bits in the input string $x$. Imagine fixing these bits in $x$ to achieve the minimum value of the parameter $s$. Denote this minimum value of $s$ by $v$. (We do not need for $v$ to be efficiently computable in any sense.) We get a nonuniform $\mathrm{NC}^{0}$ reduction from PARITY on $n-O(\log n) \geq n / 2$ bit strings to $\mathrm{MCSP}^{A}$ with the size parameter fixed to the value $v$.

- Claim 22. For any language $A$ and any $0 \leq v \leq m, \operatorname{MCSP}^{A}$ on inputs $f \in\{0,1\}^{m}$, with the size parameter fixed to $v$, is solved by a DNF formula of size $O\left(m \cdot 2^{v^{2} \log v}\right)$.

Proof of Claim 22. Each $A$-oracle circuit of size $v$ on $\log m$ inputs can be described by a binary string of length at most $O\left(v^{2} \log v\right)$, since each of $v$ gates has at most $v$ inputs. Thus, there are at most $2^{O\left(v^{2} \log v\right)}$ Boolean functions on $\log m$ inputs that are computable by $A$-oracle circuits of size at most $v$. Checking if any one of these truth tables equals to the input truth table $f$ can be done by a DNF, where we take an OR over all easy functions, and for each easy function we use an AND gate to check equality to the input $f$. 
We conclude that PARITY on $n / 2$-bit strings is solvable by $\mathrm{AC}^{0}$ circuits of depth 3 and size $O\left(m \cdot 2^{v^{2} \log v}\right)$. Indeed, each bit of the truth table $f$ is computable by an $\mathrm{NC}^{0}$ circuit, and hence by a DNF (and a CNF) of constant size. Plugging in these DNFs (or CNFs) for the bits of $f$ into the DNF formula from Claim 22 yields the required depth-3 $\mathrm{AC}^{0}$ circuit for PARITY on inputs of length at least $n / 2$.

Next, since PARITY on $m$-bit strings requires depth-3 $\mathrm{AC}^{0}$ circuits of size at least $2^{\Omega(\sqrt{m})}[10]$, we get that $v \geq n^{1 / 5}$. Hence, on input $0^{n}$, our uniform $\mathrm{NC}^{0}$ reduction produces $(f, s)$ where $f$ is the truth table of a Boolean function on $r$-bit inputs that has $A$-oracle circuit complexity at least $v \geq n^{1 / 5} \geq 2^{\epsilon r}$, for some $\epsilon>0$.

Finally, since the $N C^{0}$ reduction is (uniform $A C^{0}$ )-uniform, we get that the Boolean function whose truth table is $f$ is computable in LTH.

Proof of Theorem 20. Towards a contradiction, suppose that $\mathrm{MCSP}^{A}$ is $\mathrm{TC}^{0}$-hard under uniform $\mathrm{AC}^{0}$ reductions. Then, by Lemma 21, there is a language $L \in \mathrm{PH}$ that requires $A$-oracle circuit complexity $2^{\Omega(n)}$ almost everywhere. However, since $A$ is $\mathrm{PH}$-hard under $\mathrm{P} /$ poly reductions, we get that $L \in \mathrm{SIZE}^{A}[$ poly]. A contradiction.

- Corollary 23. $\mathrm{MCSP}^{\oplus} \mathrm{P}$ is not $\mathrm{TC}^{0}$-hard under uniform $\mathrm{AC}^{0}$ reductions.

- Corollary 24. Suppose that, for some oracle $A, \mathrm{MCSP}^{A}$ is $\mathrm{TC}^{0}$-hard under uniform $\mathrm{AC}^{0}$ reductions. Then $\mathrm{NP}^{A} \nsubseteq \mathrm{SIZE}^{A}[$ poly].

- Remark. Murray and Williams [13] prove results similar to (and implied by) our Lemma 21 and Corollary 24 for the case of the empty oracle $A=\emptyset$. Namely, they show that if MCSP is

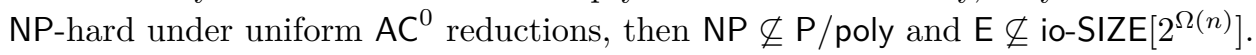

Finally, we observe that the ideas in our proof of Lemma 21 yield an alternate proof of the result by Murray and Williams [13] that PARITY is not reducible to MCSP via "local" $O\left(n^{1 / 2-\epsilon}\right)$-time reductions. We prove the version for polylogtime-uniform $\mathrm{NC}^{0}$ reductions, but the same argument applies also to the "local" reductions of [13].

- Theorem 25 ([13]). There is no polylogtime-uniform $\mathrm{NC}^{0}$ reduction from PARITY to MCSP.

Proof. Suppose there is such a reduction. Similarly to the proof of Lemma 21, we conclude that this $\mathrm{NC}^{0}$ reduction maps $0^{n}$ to an MCSP instance $(f, s)$ where $f$ is the truth table of a Boolean function on $r:=O(\log n)$ inputs that requires exponential circuit size $s \geq 2^{\Omega(r)}$. On the other hand, since our $\mathrm{NC}^{0}$ reduction is polylogtime-uniform, the Boolean function with the truth table $f$ is computable in $\mathrm{P}$, and hence in SIZE[poly]. A contradiction.

\subsection{Gap MCSP}

For $0<\epsilon<1$, we consider the following gap version of MCSP, denoted $\epsilon$-gap MCSP: Given $(f, s)$, output 'Yes' if $f$ requires circuits of size at least $s$, and output 'No' if $f$ can be computed by a circuit of size at most $(1-\epsilon) s$.

For $\alpha: \mathbb{N} \rightarrow \mathbb{R}^{+}$, call a mapping $R:\{0,1\}^{n} \rightarrow\{0,1\}^{m} \alpha$-stretching if $m \leq \alpha(n) \cdot n$. We will prove that there is no $n^{\delta}$-stretching nonuniform AC ${ }^{0}$ reduction from PARITY to $\epsilon$-gap $\mathrm{MCSP}$, for certain parameters $0<\epsilon, \delta<1$. First, we rule out nonuniform $\mathrm{NC}^{0}$ reductions.

- Theorem 26. For every $n^{-1 / 6}<\epsilon<1$ and for every constant $\delta<1 / 30$, there is no $n^{\delta}$-stretching (nonuniform) NC ${ }^{0}$ reduction from PARITY to $\epsilon$-gap MCSP. 
Proof. Towards a contradiction, suppose there is an $n^{\delta}$-stretching $\mathrm{NC}^{0}$ reduction from PARITY on inputs $x \in\{0,1\}^{n}$ to $\epsilon$-gap MCSP instances $(f, s)$. Fix to zeros all $O(\log n)$ bit positions in the string $x$ that determine the value of the size parameter $s$. As in the proof of Lemma 21, we get an $\mathrm{NC}^{0}$ reduction from PARITY on at least $n / 2$ bits $y$ to the $\epsilon$-gap MCSP instance with the size parameter fixed to some value $v \geq n^{1 / 5}$.

By our assumption, $|f| \leq n \cdot n^{\delta}$. Since each bit of $f$ is computable by an $\mathrm{NC}^{0}$ circuit, we get that each bit of $f$ depends on at most $c$ bits in the input $y$. The total number of pairs $(i, j)$ where $f_{i}$ depends on bit $y_{j}$ is at most $c \cdot|f|$. By averaging, there is a bit $y_{j}, 1 \leq j \leq n / 2$, that influences at most $c|f| /(n / 2) \leq 2 c n^{\delta}$ bit positions in the string $f$.

Fix $y$ so that all bits are 0 except for $y_{j}$ (which is set to 1 ). This $y$ is mapped by our $N C^{0}$ reduction to the truth table $f^{\prime}$ that is computable by a circuit of size at most $(1-\epsilon) v$. On the other hand, flipping the bit $y_{j}$ to 0 forces the reduction to output a truth table $f^{\prime \prime}$ of circuit complexity at least $v$. But, $y_{j}$ influences at most $2 c n^{\delta}$ positions in $f^{\prime}$, and so the circuit complexity of $f^{\prime \prime}$ differs from that of $f^{\prime}$ by at most $O\left(n^{\delta} \log n\right)$ gates (as we can just construct a "difference" circuit of that size that is 1 on the at most $2 \mathrm{cn} \delta$ affected positions of $\left.f^{\prime}\right)$. We get $\epsilon v \leq O\left(n^{\delta} \log n\right)$, which is impossible when $\delta<1 / 30$.

Now we extend Theorem 26 to the case of nonuniform $\mathrm{AC}^{0}$ reductions.

- Theorem 27. For every $n^{-1 / 7}<\epsilon<1$ and for every constant $\delta<1 / 31$, there is no $n^{\delta}$-stretching (nonuniform) $\mathrm{AC}^{0}$ reduction from PARITY to $\epsilon$-gap MCSP.

Proof. Towards a contradiction, suppose there is a $n^{\delta}$-stretching $\mathrm{AC}^{0}$ reduction from PARITY on $n$-bit strings to the $\epsilon$-gap MCSP. We will show that this implies the existence of an $\mathrm{NC}^{0}$ reduction with parameters that contradict Theorem 26 above.

- Claim 28. For every constant $\gamma>0$, there exist a constant $a>0$ and a restriction of our $\mathrm{AC}^{0}$ circuit satisfying the following: (1) each output of the restricted circuit depends on at most a inputs, and (2) the number of unrestricted variables is at least $n^{1-\gamma}$.

Proof of Claim 28. Recall that a random $p$-restriction of $n$ variables $x_{1}, \ldots, x_{n}$ is defined as follows: for each $1 \leq i \leq n$, with probability $p$, leave $x_{i}$ unrestricted, and with probability $1-p$, set $x_{i}$ to 0 or 1 uniformly at random. By Håstad's Switching Lemma [10], the probability that a given CNF on $n$ variables with bottom fan-in at most $t$ does not become a decision tree of depth at most $r$ after being hit with a random $p$-restriction is at most $(5 p t)^{r}$.

For an $\mathrm{AC}^{0}$ circuit of size $n^{k}$ and depth $d$, set $p:=(5 a)^{-1} n^{-2 k / a}$ for some constant $a>0$ to be determined. Applying this random $p$-restriction $d$ times will reduce the original circuit to a decision tree of depth $a$ with probability at least $1-d n^{k}(5 p a)^{a}>3 / 4$. The expected number of unrestricted variables at the end of this process is $p^{d} n \geq \Omega\left(n / n^{2 k d / a}\right)=\Omega\left(n / n^{\gamma^{\prime}}\right)$, for $\gamma^{\prime}:=2 k d / a$. By Chernoff bounds, the actual number of unrestricted variables is at least $1 / 2$ of the expectation with probability at least $3 / 4$.

Thus, with probability at least $1 / 2$, we get a restriction that makes the original $\mathrm{AC}^{0}$ circuit into an $\mathrm{NC}^{0}$ circuit on at least $n / n^{2 \gamma^{\prime}}$ variables, where each output of the new circuit depends on at most $a$ input variables. Setting $\gamma:=2 \gamma^{\prime}$, we get that $a=(4 k d) / \gamma$.

We get an $\mathrm{NC}^{0}$ reduction from PARITY on $n^{\prime}:=n^{1-\gamma}$ variables to $\epsilon$-gap MCSP. This reduction is at most $\left(n^{\prime}\right)^{(\delta+\gamma) /(1-\gamma)}$-stretching. Choose $0<\gamma<(1 / 31)^{2}$ so that $(\delta+\gamma) /(1-$ $\gamma)<1 / 30$, and $\epsilon>n^{-1 / 7}>\left(n^{\prime}\right)^{-1 / 6}$. Finally, appeal to Theorem 26 for contradiction. 


\section{Generalizations}

Theorem 12 gives consequences of MCSP being hard for $P$. The property of $P$ that is exploited in the proof is that the polynomial hierarchy collapses to $\mathrm{P}$ if NP $=\mathrm{P}$. (This is required, so that we can efficiently a circuit that computes bits of the reduction, knowing only that it is in the polynomial hierarchy.)

The next theorem formalizes this observation:

- Theorem 29. Let $\mathcal{C}$ be any class such that if $\mathrm{NP}=\mathcal{C}$, then $\mathrm{PH}=\mathcal{C}$. If there is a set $A \in \mathrm{PH}$ that is hard for $\mathcal{C}$ under $\leq_{T}^{p}$ reductions such that $\mathrm{MCSP}^{A}$ (or $\mathrm{MKTP}^{A}$ ) is hard for $\mathcal{C}$ under uniform $\mathrm{AC}^{0}$ reductions, then $\mathrm{NP} \neq \mathcal{C}$.

- Corollary 30. Let $A$ be any set in the polynomial hierarchy. If $\mathrm{MCSP}^{A}$ (or $\mathrm{MKTP}^{A}$ ) is hard for $\mathrm{AC}^{0}[6]$ under $\mathrm{AC}^{0}$ reductions, then $\mathrm{AC}^{0}[6] \neq \mathrm{NP}$.

Recall that SZK denotes the class of languages with Statistical Zero-Knowledge proofs.

- Corollary 31. Let $A$ be any set in the polynomial hierarchy that is hard for SZK under $\leq_{T}^{p}$ reductions. If $\mathrm{MCSP}^{A}$ is hard for $\mathrm{SZK}$ under $\mathrm{AC}^{0}$ reductions, then $\mathrm{SZK} \neq \mathrm{NP}$.

Proof. SZK is closed under complementation [14]. Thus if NP is equal to the class of languages in SZK, then coNP $=\mathrm{NP}=\mathrm{SZK}$ and $\mathrm{PH}$ collapses to SZK. Thus SZK satisfies the hypothesis of Theorem 29.

Similarly, we can state the following theorem about $\mathrm{TC}^{0}$ reductions.

- Theorem 32. Let $\mathcal{C}$ be any class such that if $\mathrm{PP}=\mathcal{C}$, then $\mathrm{CH}=\mathcal{C}$. If there is a set $A \in \mathrm{CH}$ that is hard for $\mathcal{C}$ under $\leq_{T}^{p}$ reductions such that $\mathrm{MCSP}^{A}$ (or $\mathrm{MKTP}^{A}$ ) is hard for $\mathcal{C}$ under uniform $\mathrm{TC}^{0}$ reductions, then $\mathrm{PP} \neq C$.

Fenner, Fortnow, and Kurtz [8] introduced several complexity classes, including SPP and WPP that are "low for PP", in the sense that PP $=P P^{S P P}=P P W P P$. Thus we obtain the following corollary:

- Corollary 33. Let $A$ be any set in the counting hierarchy that is hard for WPP under $\leq_{T}^{p}$ reductions. If $\mathrm{MCSP}^{A}$ is hard for WPP (or SPP) under uniform $\mathrm{TC}^{0}$ reductions, then $\mathrm{WPP} \neq \mathrm{PP}$ (respectively $\mathrm{SPP} \neq \mathrm{PP})$.

\section{Discussion}

The contrast between Theorem 12 and Corollary 18 is stark. Theorem 12 obtains a very unsurprising consequence from the assumption that MCSP is hard for $\mathrm{P}$ under a very restrictive class of reductions, while Corollary 18 obtains a very unlikely collapse from the assumption that the apparently much harder problem MCSPQBF is hard for P under a much less restrictive class of reductions. Yet, the absence of any known efficient reduction from MCSP to MCSPQBF means that we have been unable to obtain any unlikely consequences by assuming that MCSP is hard for $\mathrm{P}$. We believe that it should be possible to provide evidence that MCSP is not hard for $\mathrm{P}$, and we pose this as an open question for further research.

Acknowledgments This research was supported in part by NSF grants CCF-1064785 and CCF-1423544, and by an NSERC Discovery Grant. Some of this work was carried out at the 2014 Dagstuhl Workshop on Algebra in Computational Complexity (Dagstuhl Seminar 14391). We also acknowledge helpful discussions with Ryan Williams, Chris Umans, Manindra Agrawal, and Mitsunori Ogihara. 


\section{References}

1 Manindra Agrawal. The isomorphism conjecture for constant depth reductions. Journal of Computer and System Sciences, 77(1):3-13, 2011.

2 Manindra Agrawal. Personal Communication, 2014.

3 Eric Allender, Harry Buhrman, Michal Kouckỳ, Dieter van Melkebeek, and Detlef Ronneburger. Power from random strings. SIAM Journal on Computing, 35(6):1467-1493, 2006.

4 Eric Allender and Bireswar Das. Zero knowledge and circuit minimization. In Mathematical Foundations of Computer Science (MFCS), volume 8635 of Lecture Notes in Computer Science, pages 25-32. Springer, 2014.

5 Eric Allender and Vivek Gore. On strong separations from $\mathrm{AC}^{0}$. In Jin-Yi Cai, editor, Advances in Computational Complexity Theory, volume 13 of DIMACS Series in Discrete Mathematics and Theoretical Computer Science, pages 21-37. AMS Press, 1993.

6 Eric Allender, Michal Koucký, Detlef Ronneburger, and Sambuddha Roy. The pervasive reach of resource-bounded Kolmogorov complexity in computational complexity theory. Journal of Computer and System Sciences, 77:14-40, 2010.

7 José L Balcázar, Antoni Lozano, and Jacobo Torán. The complexity of algorithmic problems on succinct instances. In Computer Science, pages 351-377. Springer, 1992.

8 Stephen A. Fenner, Lance Fortnow, and Stuart A. Kurtz. Gap-definable counting classes. Journal of Computer and System Sciences, 48(1):116-148, 1994.

9 Hana Galperin and Avi Wigderson. Succinct representations of graphs. Information and Control, 56(3):183-198, 1983.

10 Johan Håstad. Almost optimal lower bounds for small depth circuits. In Proceedings of the Eighteenth Annual ACM Symposium on Theory of Computing, pages 6-20, 1986.

11 Valentine Kabanets and Jin-Yi Cai. Circuit minimization problem. In Proceedings of the thirty-second annual ACM symposium on Theory of computing, pages 73-79. ACM, 2000.

12 Leonid Levin. Randomness conservation inequalities; information and independence in mathematical theories. Information and Control, 61:15-37, 1984.

13 Cody Murray and Ryan Williams. On the (non) NP-hardness of computing circuit complexity. In Electronic Colloquium on Computational Complexity (ECCC), 2014. TR14-164.

14 Tatsuaki Okamoto. On relationships between statistical zero-knowledge proofs. Journal of Computer and System Sciences, 60(1):47-108, 2000.

15 Christos H. Papadimitriou. Computational complexity. John Wiley and Sons Ltd., 2003.

16 Christos H. Papadimitriou and Mihalis Yannakakis. A note on succinct representations of graphs. Information and Control, 71(3):181-185, 1986.

17 Boris A. Trakhtenbrot. A survey of Russian approaches to perebor (brute-force searches) algorithms. IEEE Annals of the History of Computing, 6(4):384-400, 1984.

18 Klaus W Wagner. The complexity of combinatorial problems with succinct input representation. Acta Informatica, 23(3):325-356, 1986.

19 Ryan Williams. http://cstheory.stackexchange.com/questions/10320/succinct-problems-inmathsfp/10546\#10546, 2012. 